\title{
The corpus callosum in first episode schizophrenia: a diffusion tensor imaging study
}

\author{
G Price, M S Bagary, M Cercignani, D R Altmann, M A Ron
}

J Neurol Neurosurg Psychiatry 2005;76:585-587. doi: 10.1136/jnnp.2004.042952

\begin{abstract}
Background: Neuropathological and imaging studies suggest that corpus callosum abnormalities (CC) are present in schizophrenia, but it remains to be determined whether these abnormalities are present at illness onset. Diffusion tensor imaging (DTI), which is more sensitive than conventional magnetic resonance imaging (MRI) in detecting subtle structural changes in the organisation and integrity of white matter tracts, is an ideal tool to investigate this question.

Objective: To determine whether CC abnormalities are present at illness onset in schizophrenia.

Methods: Twenty patients (14 men, six women) with first episode schizophrenia and 29 controls (11 men, 18 women) were studied. Both high resolution volumetric $\mathrm{Tl}$-weighted images and DTI were acquired. Regions of interest (ROI) were placed in the splenium and genu of the CC and fractional anisotropy (FA) and diffusivity (D) measured.

Results: No differences in FA or D were detected in these regions between patients and controls. In women, irrespective of group membership, FA was significantly lower and there was a trend for $D$ to be higher than in men, indicating less barriers to diffusion in females.

Conclusion: The negative findings of this study suggest that in the early stages of schizophrenia there is no disruption to the integrity of the CC and raise the possibility that the neuropathological abnormalities may appear later and be progressive, at least in some patients.
\end{abstract}

$\mathrm{D}$ ifferences in the size of the corpus callosum (CC) between individuals with chronic schizophrenia and controls were first reported in a post mortem study by Rosenthal and Bigelow, ${ }^{1}$ who showed the CC to be wider in schizophrenic individuals. However, magnetic resonance imaging (MRI) studies have yielded contradictory results. In a review of 27 MRI studies of the CC in schizophrenia, only 17 studies were found to have reported differences between the CC in schizophrenic individuals and controls, but variation in methods was cited as contributing to the inconsistent findings. There are few neuropathological studies of the CC in schizophrenia. Nasrallah et $a l^{3}$ found more gliosis in the CC of individuals with late onset schizophrenia compared with early onset schizophrenia and controls. A greater reduction in the total number of fibres in all regions of the CC, except in the rostrum, has also been reported in females compared with males with schizophrenia and this difference was reversed in controls. ${ }^{4}$

Abnormalities in the CC can lead to disordered transfer of information between the two hemispheres ${ }^{5}$ and may explain some of the symptoms and cognitive abnormalities encountered in schizophrenia. ${ }^{6}$ Functional abnormalities of the CC in schizophrenia have been reported in behavioural studies. Experimental tasks requiring transfer of information between the hemispheres through the CC show evidence for dysfunctional interhemispheric connectivity in patients with schizophrenia. ${ }^{7}$ There is no information as to whether any of these abnormalities in the CC are present early in the disease.

Diffusion tensor imaging (DTI) is of particular interest in this regard as it appears to be sensitive to the presence of subtle white matter abnormalities in the absence of volumetric changes detectable by conventional MRI. DTI provides information about white matter tracts and their organisation based on water diffusion. Diffusion in white matter is more restricted across a fibre than along its axis. This property is termed diffusion anisotropy. Mean diffusivity (D) is a directionally independent measure of the average diffusivity, and fractional anisotropy (FA) is a measure of the degree of anisotropy and thus of organisation of white matter tracts. Increased D may result from demyelination, axon loss, or oedema, ${ }^{8}$ while reduced FA indicates disruption of the organisation of fibre tracts. ${ }^{8}$

In a cross-sectional DTI study of chronic schizophrenia, our group successfully detected significant differences in the splenium of the CC of patients compared with healthy controls suggesting focal disruption of commissural connectivity. ${ }^{9}$ We present here the results of a study of patients with first episode schizophrenia, using similar methodology, to determine whether CC abnormalities are already present in the early stages of the illness.

\section{METHODS}

\section{Subjects}

A total of 20 patients (14 men, six women; mean age 24.95 years, range 18-49) who fulfilled the DSM-IV diagnosis of schizophrenia were recruited from mental health services in west London and investigated within one month of presentation to these services. The control group consisted of 29 healthy volunteers (11 men, 18 women; mean age 28.06 years, range $20-40$ ). Individuals with a history of head injury leading to unconsciousness, systemic illness, or substance misuse at the time of the study were excluded from the study. Antipsychotic medication was prescribed to 16 patients (14 atypical). Three patients and two controls were left handed..$^{10}$ The relevant ethics committees approved the study and all subjects provided written informed consent. All subjects had participated in an imaging study of volumetric MRI and magnetisation transfer imaging. ${ }^{11}$

\section{Imaging}

DTI was performed on a Signa 1.5 Tesla scanner (General Electric, Milwaukee, WI) with a standard quadrature head coil. A localising scan was acquired. Diffusion weighted echo planar images (DW-EPI) were acquired in the axial plane

Abbreviations: CC, corpus callosum; D, mean diffusivity; DTI, diffusion tensor imaging; FA, fractional anisotropy; MRI, magnetic resonance imaging; ROl, region of interest 


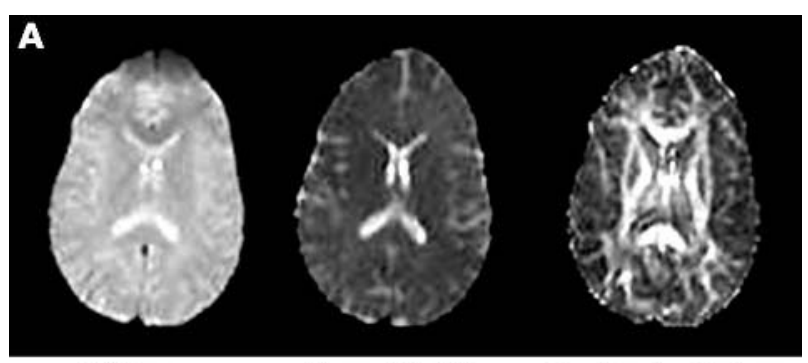

b0 image Mean diffusivity Fractional anisotropy

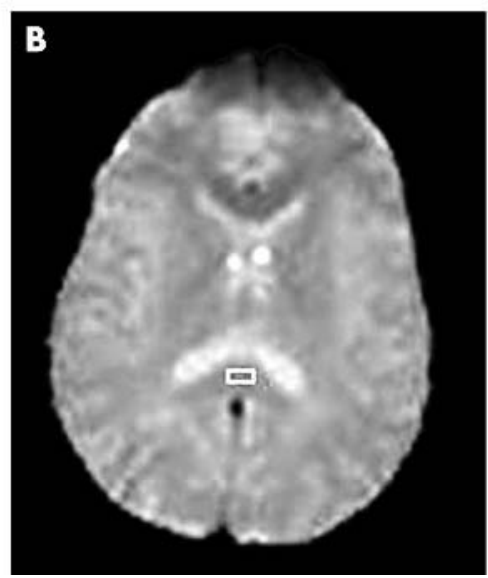

Figure 1 (A) Axial b0 and diffusion tensor images showing the corpus callosum with (B) a $28 \mathrm{~mm}^{2}$ region of interest in the splenium.

(TE $=78 \mathrm{~ms}, 96 \times 96$ matrix, field of view $24 \mathrm{~cm} \times 24 \mathrm{~cm}$, slice thickness $=5 \mathrm{~mm}$ ). Gradients for diffusion sensitisation were applied in seven non-collinear directions. Each direction was sampled using four gradient strengths corresponding to $\mathrm{b}$ values from 0 to $700 \mathrm{~s} / \mathrm{mm}^{2}$. Images were acquired in three sets of interleaved slices. Five acquisitions of each set were performed and averaged after magnitude reconstruction, improving signal to noise ratio. Image acquisition was cardiac gated with a peripheral pulse oximeter. After affine registration of the DW-EPI with the corresponding b0 images, the diffusion tensor was estimated, ${ }^{12}$ and maps of $\mathrm{D}$ and $\mathrm{FA}^{13}$ were calculated.

The whole of the CC was contained within 21 contiguous slices. Regions of interest (ROIs) of standard size $\left(28 \mathrm{~mm}^{2}\right)$ were placed on the b0 image containing the genu and splenium on the slice showing maximal CC thickness (fig 1). Slices contiguous with the one selected were examined to avoid the partial volume effects of cerebrospinal fluid (CSF). To avoid placement bias the ROIs were automatically transferred to the inherently coregistered corresponding diffusion maps. A second rater assessed 24 randomly chosen subjects (10 with first episode schizophrenia and 14 controls). The percentage of overlapping voxels in the two ROIs was used as a test of interrater reliability, and this was calculated at $64 \%$.

\section{Statistical analysis}

Regressions were carried out for D and FA on a patient versus control group indicator. Age, gender, and handedness were used as covariates to compare patients and controls adjusted for possible confounding; unadjusted comparisons were made with $t$ tests. These models also gave estimates of gender differences. There was no evidence from residuals of any serious non-normality or heteroscedasticity. Statistical significance is reported at the $5 \%$ level.

\section{RESULTS}

The gender distribution was different between the two groups (38\% of controls were men $v 70 \%$ of patients), but age distribution was similar. There were no significant differences in D and FA between the patients and controls, adjusting for age, gender, and handedness. Table 1 shows unadjusted and adjusted results for FA and D in both regions (genu and splenium). In the genu, a gender effect was present irrespective of group membership, with the women having significantly lower FA, by -0.077 (95\% confidence interval (CI) -0.029 to $-0.124 ; \mathrm{p}=0.002$ ) and borderline significantly higher D, by 0.050 (95\% CI -0.002 to 0.101 ; $\mathrm{p}=0.058$ ) than men, suggesting fewer barriers to diffusivity. These results were unchanged when group was adjusted for.

\section{DISCUSSION}

The present study failed to demonstrate abnormalities in the CC of patients with first episode schizophrenia in contrast with our previous findings in patients with chronic schizophrenia, using a similar technique. ${ }^{9}$ We chose to examine the CC because it is the major fibre tract connecting the two hemispheres, and it is easily identifiable, allowing ROIs to be reliably placed with minimal intersubject variability in the directionality of its fibres. The contrasting findings of our two studies suggest that in the early stages of schizophrenia, interhemispheric connectivity is normal or that pathological changes are too subtle to be detected with this technique, and that neuropathological changes in the CC may be a result of a progressive and chronic illness, at least in some patients. It is also possible that CC abnormalities may have been present at the beginning of the illness only in those patients destined to follow a chronic trajectory and our small sample prevented their detection. On the other hand, it is unlikely that those willing to take part in the study had a more benign illness, as only one of those deemed to be suitable refused to participate. It is also unlikely that the contrasting findings of our studies

Table 1 Mean diffusivity and fractional anisotropy in the splenium and genu

\begin{tabular}{|c|c|c|c|c|}
\hline & $\begin{array}{l}\text { Patients with schizophrenia } \\
n=20 \\
\text { Mean (SD) }\end{array}$ & $\begin{array}{l}\text { Controls } \\
n=29 \\
\text { Mean (SD) }\end{array}$ & $\begin{array}{l}\text { Patient-control difference unadjusted } \\
\text { ( } 95 \% \mathrm{Cl} \text {; p value) }\end{array}$ & $\begin{array}{l}\text { p value after } \\
\text { adjustment* }\end{array}$ \\
\hline D (splenium) & $0.859(0.132)$ & $0.873(0.148)$ & $\begin{array}{l}-0.015 \\
(-0.097 \text { to } 0.068 ; p=0.73)\end{array}$ & 0.91 \\
\hline D (genu) & $0.887(0.086)$ & $0.890(0.097)$ & $\begin{array}{l}-0.003 \\
(-0.057 \text { to } 0.051 ; p=0.91)\end{array}$ & 0.82 \\
\hline FA (splenium) & $0.748(0.117)$ & $0.736(0.085)$ & $\begin{array}{l}0.012 \\
(-0.046 \text { to } 0.070 ; p=0.67)\end{array}$ & 0.93 \\
\hline FA (genu) & $0.699(0.083)$ & $0.681(0.096)$ & $\begin{array}{l}0.018 \\
(-0.035 \text { to } 0.071 ; p=0.50)\end{array}$ & 0.76 \\
\hline
\end{tabular}

*Adjusted for gender, age, and handedness.

$\mathrm{Cl}$, confidence interval; $\mathrm{D}$, mean diffusivity $\left(\times 10^{3} \mathrm{~mm}^{2} / \mathrm{s}\right)$; FA, fractional anisotropy (dimensionless units). 
are due to interrater variability, as the same size ROIs and strict criteria for their placement were used in both studies and interrater reliability was high.

Previous studies using DTI in schizophrenia have reported conflicting results when exploring the CC and other white matter regions. Thus, differences between patients and controls have been reported by some, ${ }^{9-18}$ but not by others. ${ }^{19}{ }^{20}$ Examination of other areas of white matter in our study may have revealed differences between patients and controls, but we believe that ROI methodology is not well suited to examine areas of white matter with weaker directionality than the CC.

Recent conventional MRI studies of the CC using differing methodologies have suggested reductions in size ${ }^{21}$ and shape differences $^{22}$ in patients with first episode schizophrenia compared with controls. However, other studies have not confirmed these findings. ${ }^{23}{ }^{24}$ Different methods and patient populations may explain these differences.

The differences in the FA and D in the genu of the CC in the women in our study, irrespective of group membership, are similar to those reported in normal subjects by Westerhausen et al. ${ }^{25}$ In the light of Highley et al's, ${ }^{4}$ neuropathological report, both Westerhausen's and our findings are likely to be due to the greater thickness of myelin sheaths and smaller interfibre spaces in men that would result in comparatively fewer barriers to diffusivity and hence lower FA and higher D in women. The small number of women in our first episode schizophrenia group prevented us from determining with any degree of certainty whether the same pattern of DTI differences between males and females was also present in our patients.

\section{ACKNOWLEDGEMENTS}

We are grateful to members of the NMR Unit and Dr Dominique Fluegel for doing the inter-rater reliability. We also wish to thank all the subjects who participated in this study.

\section{Authors' affiliations London, UK \\ Competing interests: none declared M.Ron@ion.ucl.ac.uk \\ Received 8 April 2004 \\ Revised version received 29 July 2004 \\ Accepted 5 August 2004}

G Price, M S Bagary, M Cercignani, D R Altmann, M A Ron, Institute of Neurology, University College London, London, UK

D R Altmann, London School of Hygiene and Tropical Medicine,

This study was supported by a grant from the Wellcome Trust.

Correspondence to: Professor M A Ron, Institute of Neurology, University College London, Queen Square, London, WCIN 3BG, UK;

\section{REFERENCES}

1 Rosenthal R, Bigelow LB. Quantitative brain measurements in chronic schizophrenia. Br J Psychiatry 1972;121:259-64.

2 Shenton ME, Dickey CC, Frumin M, et al. A review of MRI findings in schizophrenia. Schizophr Res 2001;49:1-52.

3 Nasrallah HA, McCalley-Whitters M, Bigelow LB, et al. A histological study of the corpus callosum in chronic schizophrenia. Psychiatry Res 1983;8:251-60.

4 Highley JR, Esiri MM, McDonald B, et al. The size and fibre composition of the corpus callosum with respect to gender and schizophrenia:a post-mortem study. Brain 1999;12:99-110.

5 O'Shea RP, Corballis PM. Binocular rivalry in split-brain observers. J Vis 2003;3:610-15.

6 Crow TJ. Schizophrenia as a transcallosal misconnection syndrome. Schizophr Res 1998;30:111-14.

7 Mor B, Friedmann P, Cohen R, et al. Interhemispheric cooperation during word processing: evidence for callosal transfer dysfunction in schizophrenic patients. Schizophr Res 2000;46:231-9.

8 Werring DJ, Clark CA, Barker GJ, et al. Diffusion tensor imaging of lesions and normal-appearing white matter in multiple sclerosis. Neurology 1999:52:1626-32.

9 Foong J, Maier M, Clark CA, et al. Neuropathological abnormalities of the corpus callosum in schizophrenia: a diffusion tensor imaging study. J Neurol Neurosurg Psychiatry 2000;68:242-4.

10 Annett MA. A classification of hand preference by association analysis. Br J Psychology 1970;61:303-21.

11 Bagary MS, Symms MR, Barker GJ, et al. Gray and white matter abnormalities in first-episode schizophrenia inferred from magnetisation transfer imaging. Arch Gen Psychiatry 2003;60:779-88.

12 Basser PJ, Mattielo J, Le Bihan D. Estimation of the effective self-diffusion tensor from the NMR spin echo. J Magn Reson B 1994; 103:247-54.

13 Pierpaoli C, Basser PJ. Towards a quantitative assessment of diffusion anisotropy. Magn Reson Med 1996;36:893-906.

14 Bauchbaum MS, Tang CY, Peled S, et al. MRI white matter diffusion anisotropy and PET metabolic rate in schizophrenia. Neuroreport 1998; 16:425-30

15 Lim KO, Hedehus M, Moseley M, et al. Compromised white matter tract integrity in schizophrenia inferred from diffusion tensor imaging. Arch Gen Psychiatry 1999;56:367-74.

16 Agartz I, Andersson JLR, Skare S. Abnormal white mater in schizophrenia: a diffusion tensor imaging study. Neuroreport 2001;12:2251-4.

17 Kubiki M, Westin CF, Maier SE, et al. Uncinate fasciculus findings in schizophrenia: a magnetic resonance diffusion tensor imaging study. Am J Psychiatry 2002; 159:813-20.

18 Burns J, Job D, Bastin ME, et al. Structural disconnectivity in schizophrenia, a diffusion tensor magnetic resonance imaging study. $\mathrm{Br} J$ Psychiatry 2003;182:439-43

19 Steel RM, Bastin ME, McConnell S, et al. Diffusion tensor imaging (DTI) and proton magnetic resonance spectroscopy (1H MRS) in schizophrenic subjects and controls. Psychiatry Res 2001;106:161-70.

20 Foong J, Symms MR, Barker GJ, et al. Investigating regional white matter in schizophrenia using diffusion tensor imaging. Neuroreport 2002;13:333-6.

21 Bachmann S, Pantel J, Flender A, et al. Corpus callosum in first-episode patients with schizophrenia-a magnetic resonance imaging study. Psychol Med 2003;33:1019-27.

22 Frumin $M$, Golland $P$, Kikinis R, et al. Shape differences in the corpus callosum in first-episode schizophrenia and first-episode psychotic affective disorder. Am J Psychiatry 2002;159:866-8.

23 Woodruff PWR, Philips ML, Rushe T, et al. Corpus callosum size and function in schizophrenia. Schizophr Res 1997;23:189-96.

24 Meisenzahl EM, Frodl T, Greiner J, et al. Corpus callosum size in schizophrenia-a magnetic resonance imaging analysis. Eur Arch Psychiatry Clin Neurosci 1999;249:305-12.

25 Westerhausen R, Walter C, Kreuder F, et al. The influence of handedness and gender on the microstructure of the human corpus callosum: a diffusion-tensor magnetic resonance imaging study. Neurosci Lett 2003;13:99-102. 